\title{
Dispersion behaviour of solid particles in flow-injection analysis
}

\author{
Marcel Hulsman, Martinus Bos, Willem E. van der Linden* \\ Laboratory of Chemical Analysis, Department of Chemical Technology, University of Twente, P.O. Box 217, \\ 7500 AE Enschede, The Netherlands
}

Received 22 November 1996; received in revised form 21 February 1997; accepted 25 February 1997

\begin{abstract}
In order to achieve a reproducible on-line pretreatment of slurry samples in flow-injection analysis the dispersion behaviour of these samples has to be examined. This paper reports the effects of flow rate and some configurations of reaction manifolds. The reaction manifolds were a straight tube and two helically coiled tubes with different aspect ratios and spatial orientation. The slurry samples consisted of spheres of different densities suspended in a Triton X-100/water solution, the particle diameters (up to $75 \mu \mathrm{m}$ ) were relatively large compared to the internal diameter of the tubing $(0.75 \mathrm{~mm})$. For the straight tube theoretical calculations were found to give a reasonable impression of the experimental flow behaviour of the spheres. Statistical moments were used to characterize the slurry peaks. For tightly coiled tubes it was found that the retention time of a slurry sample depends on the particle density. The spatial orientation of the coils mainly affects the retention of high density particles.
\end{abstract}

Keywords: Flow injection; Dispersion

\section{Introduction}

On-line pretreatment of slurry samples in flowinjection analysis (FIA) systems decreases the amount of batch-like manipulation needed prior to the analysis of solid samples. It shortens analysis times and also increases reproducibility. Therefore, direct injection of slurries is receiving an increasing interest especially in combination with microwave digestion and/or (flame) atomic absorption spectrometric detection [1-6]. On-line solid-liquid extraction of adsorbed components is another possible option. This study is part of a project to derive design rules for the automated analysis of slurry samples in FIA with a

\footnotetext{
*Corresponding author.
}

minimum of off-line sample pretreatment and with automated direct injection of slurries. FIA systems normally require completely dissolved samples. If clogging and wear can be prevented, direct injection of solid particles can be advantageous because it allows on-line sample pretreatment. A minimisation of preparation steps like drying, grinding and sieving, prior to the slurry injection is also advantageous, especially in cases when grinding is often not allowed such as the analysis of soluble, extractable or adsorbed fractions [7]. An injector for automated direct injection of slurries was reported earlier [8].

On-line pretreatment of slurry samples requires relatively long residence times [6]. Separation due to differences in retention behaviour of particles of different size and density, as e.g. in steric field flow 
fractionation (FFF) [9] or hydrodynamic chromatography [10], has to be prevented. Therefore, to ensure a reproducible pretreatment, independent of the sample composition, it is necessary to characterize the dispersion behaviour of solid particles in order to control the (mean) residence time and minimise its variance.

Statistical moments have been widely used over the last 20 years to characterize the shape of chromatographic peak profiles [11]. In FIA, moments can provide useful information about the pattern of flow [12]. Johnson et. al. [13] used statistical moments to compare the contribution of different types of sample loops to the total system dispersion. In this study statistical moments were used to characterize the slurry peaks. Detection was performed turbidimetrically as reported earlier [8]. The signal is very noisy due to the inhomogeneity of the slurries and the peak shape is not only influenced by the flow pattern but also by the sample composition. To investigate whether pretreatment of these samples can be performed reproducibly, two moments are of importance, the first normalised moment which is the mean residence time and the second centralised normalised moment which is an indication for the variation in residence time and is an equivalent of the dispersion coefficient [14]. The actual peak shape is of less importance.

This paper reports the effects of flow rate and configuration of the reaction coil on the dispersion behaviour of slurry samples. An automated injection method was used without any contact between slurry and pump. The internal diameter of the conduits throughout the system was $0.75 \mathrm{~mm}$. The investigated slurries consisted of a suspension of solid particles in an aqueous $0.1 \%$ Triton $\mathrm{X}-100$ solution. The solids were: Dowex ion-exchange particles, Glass beads and a soil sample. The results of the slurry injections were compared with Dye injections. The reaction manifolds were two helically coiled tubes with different aspect ratios, both used in a horizontal and a vertical position, and a straight tube. The coils were relatively long to create a residence time similar to that of a typical online pretreatment unit [7]. Knotting of the tubing was not considered. Haswell et. al. [1] reported for on-line microwave digestion of slurry samples local heating and eventual blockage of the tubing in the tight bends of such configurations. To our knowledge no theore- tical relations on the retention behaviour of spheres in helically coiled tubes are available. The retention times of the slurries in a straight tube were compared with theoretical calculations based on the flow behaviour of spheres in a laminar flow regime.

\section{Theory}

\subsection{Moment analysis}

The statistical moments are defined by the following equations:

$M_{0}=\int_{0}^{\infty} h(t) \mathrm{d} t$
$M_{1}=\frac{1}{M_{0}} \int_{0}^{\infty}(t) h(t) \mathrm{d} t$
$M_{n}=\frac{1}{M_{0}} \int_{0}^{\infty}\left(t-M_{1}\right)^{n} h(t) \mathrm{d} t$

Where $h(t)$ is the height of the detector signal at time $t$. The zero-th moment $\left(M_{0}\right)$ represents the peak area. The first moment $\left(M_{1}\right)$ gives the centre of gravity or the mean of the peak profile and corresponds to the time of appearance of the peak maximum of a Gaussian peak. Moments higher than the first one are defined as central moments with their peak distribution being around the first moment. The second central moment $\left(M_{2}\right)$ is the variance in residence time and gives a measure of the spread of the peak about its centre of gravity. Both the first and the second moment are normalized and therefore independent of the sample composition and concentration.

In this study the statistical moments were calculated by direct numerical integration between peak start and peak end. This method makes no assumptions about peak shape and can be used for peaks (such as slurry peaks [8]) which cannot be fitted by Gaussian or modified Gaussian functions for which simple manual methods are available for moment calculations [15]. The direct numerical integration is straightforward and easy to implement especially in the case of computer data acquisition. However, the contribution of data points further away from the centre of gravity 
becomes quite large and any baseline drift or signal noise causes a significant inaccuracy in the calculations of the higher moments. Therefore it is important that peak start and peak end are properly assigned.

In general, the plug-like slurry response peaks cannot be fitted by (modified) Gaussian functions, therefore the first moment is taken instead of the time of appearance of the peak top when looking for the mean residence time. Peak begin and peak end are important for moment analysis but are difficult to determine for noisy signals. Therefore peak begin and peak end of the slurry peaks are determined as described earlier [8] after a 21-point cubic SavitskyGolay smoothing [16].

\subsection{Retention of spheres in laminar tube flow}

The axial velocity of a particle subjected to Poiseuille flow in a straight tube is somewhat less than the axial velocity of the undisturbed fluid at the same distance from the wall as the particle's center [17]. At a distance $r$ from the tube center the particle velocity $\nu_{\mathrm{p}}$ in axial direction is given by

$\nu_{\mathrm{p}, \mathrm{ax}}(r)=2 \bar{\nu}\left(1-\frac{r^{2}}{R^{2}}\right)-\nu_{\mathrm{p}, \mathrm{S}}$

where $\bar{\nu}$ is the mean velocity of the fluid, $R$ is the tube radius and $\nu_{\mathrm{p}, \mathrm{S}}$ the slip velocity of the particle. With $\kappa$ being the ratio of the particle radius $a$ to the tube radius $R$, for small spheres $(\kappa \ll 1)$, the slip velocity is given by the equation

$\nu_{\mathrm{p}, \mathrm{S}}=\frac{4}{3} \bar{\nu} \kappa^{2}+\frac{40}{9} \bar{\nu} \kappa^{3}\left(1+\frac{r}{R}\right) K_{D}+O\left(\kappa^{4}\right)$

with an accuracy to $O\left(\kappa^{3}\right)$ and

$K_{D}=\frac{9}{32}\left[\left(1-\frac{r}{R}\right)^{-2}-\left(1+\frac{r}{R}\right)^{-2}\right]$

Assuming that particles move at a fixed height above the bottom of a horizontal tube, the position $r$ can be calculated using the experimental retention time.

The aim of this project is to predict and control the retention times of particles of different sizes and densities. Rigid-spherical neutrally-buoyant particles carried along in Poiseuille flow, migrate to an equilibrium position (tubular pinch effect) [18]. The phenomenon of lift is of inertial origin. Lift can be viewed as arising from the pressure difference on either side of a particle which in turn arises from the difference in fluid velocity in the parabolic velocity field. The inertial lift velocity, perpendicular to the fluid motion, of a small neutrally-buoyant particle $(\kappa \ll 1)$ in a dilute suspension is

$\nu_{L i}=\frac{1}{4} \operatorname{Re}_{\mathrm{p}} \kappa^{2} U_{m} \mathrm{f}^{\mathrm{T}}(\beta)$

where $\beta$ is $r / R, U_{m}$ is the maximum fluid velocity and $\mathrm{Re}_{\mathrm{p}}$ is the Reynolds number based on the particle radius. For tube flow $\mathrm{f}^{\mathrm{T}}(\beta)$ is reported by Belfort and Nagata [19] as a 5th order polynomial form based on numerical calculations

$$
\begin{aligned}
\mathrm{f}^{\mathrm{T}}(\beta)= & 1.60152 \beta-0.860212 \beta^{2}-0.70634 \beta^{3} \\
& -2.734199 \beta^{4}+1.382202 \beta^{5}
\end{aligned}
$$

Together with the Stokes drag force, the inertial lift force for neutrally-buoyant spheres can be obtained

$F_{\mathrm{Li}}=6 \pi a \mu \cdot v_{\mathrm{Li}}$

where $\mu$ is the fluid dynamic viscosity. At the equilibrium height the inertial lift force equals zero.

Williams et al. [20] showed for flow between parallel plates (slit flow) that Eq. (9) can also be applied to non-neutrally-buoyant spheres entrained in a horizontal slit flow if an eventual additional force (e.g. the gravitational force), is working perpendicular to the fluid motion. In this case, the actual lift force together with the gravitational force determine the equilibrium height. The same reasoning can be applied to horizontal tube flow; if a particle is moving at a fixed equilibrium height the actual lift force must be equal and opposite to the gravitational force

$F_{\mathrm{L}}(\mathrm{act})=\frac{4}{3} \pi a^{3} \Delta \rho g$

where $\Delta \rho$ is the particle-fluid density difference and $g$ is the acceleration due to gravity. If a particle moves at some distance from the wall, near-wall contributions to the lift force can be neglected [20] and the actual lift force equals the inertial lift force.

$F_{\mathrm{L}}(\mathrm{act})=F_{\mathrm{Li}}$

Substituting Eq. (10) and Eq. (7) in Eq. (9) the following expression is obtained

$\mathrm{f}^{\mathrm{T}}(\beta)=\frac{2}{9} \frac{R^{2} \Delta \rho g}{\bar{\nu}^{2} a \rho_{w}}$ 
where $\rho_{w}$ is the fluid density. So, if density and radius of a particle are known, the equilibrium position $r$ can be calculated and therefore with Eq. (4) also the theoretical axial velocity can be calculated. Using this velocity and the tube length, the theoretical retention ratios can be calculated and compared with the experimentally obtained values.

\section{Experimental}

\subsection{Equipment and system control}

Fig. 1 shows the load position of the injector. Solenoid pinch valves from Bio-Chem Valve Corp. were used: P/N 100P3MP24-01B (V1 in Fig. 1), P/N 100P2NC24-01B (V2), P/N 100P2NC24-02B (V3) and $\mathrm{P} / \mathrm{N}$ 100P2NO24-01B (V4). The injector was based on the volume-based hydrodynamic injector as described earlier [8]. Besides the reduction of the internal diameter of the tubing to $0.75 \mathrm{~mm}$, two adjustments were made. First, during the load cycle slurry sample was propelled by gas pressure. The advantage is the avoidance of contact between slurry sample and a pump. Applying gas pressure makes it also possible to rinse and fill the sample loop with a relatively high flow rate. The high flow rate should improve the repeatability and the reproducibility of the sample withdrawal from a sample supply vessel [21].
Secondly, two extra carrier streams are used. During the load time particles can be retarded in the T-piece $\mathrm{Y}$, causing accumulation and eventually blockage of $\mathrm{Y}$. This is prevented by a small extra flow (C2). The problem of accumulation did not occur at T-piece X. When the valves were switched simultaneously the trapped sample volume was propelled by the carrier streams and transported through the reaction manifold to the detector. It was observed that during the injection of slurry samples with high density particles like Glass beads, a fraction of the particles was not propelled towards the reaction manifold but the particles fell down towards valve V3. A small extra carrier stream (C3) connected to the manifold between $\mathrm{Y}$ and $\mathrm{V} 3$ prevented the particles from falling. The two extra streams led to a dilution of the sample, however, each flow rate was only $5 \%$ of the total flow. The previously described injector [8] did not show any of the mentioned problems because tubes with larger internal diameters and only relatively low density particles were used.

A Gilson Minipuls 2 peristaltic pump was used for pumping the carrier solutions. Sample loop, coils and connecting lines were made of $0.75 \mathrm{~mm}$ i.d. PTFE tubing $(1.6 \mathrm{~mm}$ o.d.). The following reaction (retention) manifolds were used:

- Straight tube (horizontal).

- Helically coiled loop $\lambda=0.75 / 11\left(\mathrm{~mm} \mathrm{~mm}^{-1}\right)$.

- Helically coiled loop $\lambda=0.75 / 74\left(\mathrm{~mm} \mathrm{~mm}^{-1}\right)$.

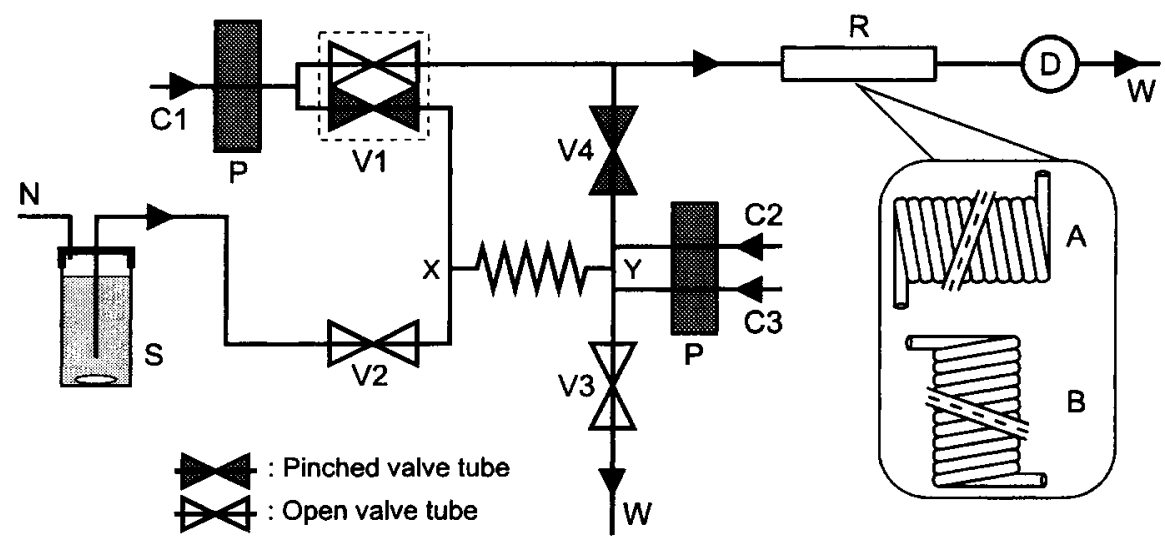

Fig. 1. Load position of the injector. S, sample vessel with screw cap and magnetic stirrer; N, gas pressure (Nitrogen, 1 atm); C1, C2 and C3, carrier; XY, injected sample volume; W, waste; P, peristaitic pump; V1, V2, V3 and V4, solenoid pinch valves; D. detector; R, reaction coil; A, horizontal orientation; $\mathrm{B}$, vertical orientation. 
$\lambda$ is the aspect ratio, i.e. tube diameter/coil diameter. The manifolds had a length of $5.5 \mathrm{~m}$. Both the coils were used in two ways, either in horizontal or in vertical orientation (Fig. 1). The sample loop was $1 \mathrm{~m}$ of $0.75 \mathrm{~mm}$ i.d. with $\lambda=0.75 / 11\left(\mathrm{~mm} \mathrm{~mm}^{-1}\right)$, the sample volume was approximately $450 \mu 1$ and the connecting tubes had a total length of $0.4 \mathrm{~m}$.

The detector was a home-built one channel version of the LED photometric detector described by Worsfold et al. [22]. The main components are an ultrabright LED $(660 \mathrm{~nm})$ and a silicon photodiode with an integral current/voltage converter and amplifier (Radio Spares 308-067). Measurements take place perpendicular to the $0.75 \mathrm{~mm}$ i.d. PTFE tubing, the width of the lightbeam was approximately $1 \mathrm{~mm}$.

A program written in $C$ was used to control valve switching, light intensity of the LED, detector offset (baseline adjustment) and the data acquisition $(100 \mathrm{~Hz})$. The computer hardware consisted of an IBM compatible PC (486/66 MHz), a Keithley interface card (DAS-1600) and a home-made passive DA/ $\mathrm{AD}$ interface.

\subsection{Chemicals}

All chemicals were of analytical grade and all solutions were prepared in Millipore Q2 water. Slurry samples consisted of particles in an aqueous $0.1 \%$ ( $\mathrm{v} /$ v) Triton $\mathrm{X}-100$ solution. The particles, diameter range, density and concentrations used were:

- Dowex 50w-x8, 37-75 $\mu \mathrm{m}, \rho=1.2 \mathrm{~g} \mathrm{~m}^{-1}$, $20 \mathrm{mg} \mathrm{ml}^{-1}$; (further referred to as Dowex).

- Glass beads, $10-60 \mu \mathrm{m}, \rho=2.45 \mathrm{~g} \mathrm{ml}^{-1}$, $6 \mathrm{mg} \mathrm{ml}^{-1}$; (Glass).

- Contaminated land soil (sampled from a known site); concentration: $12 \mathrm{mg} \mathrm{ml}^{-1}$; (Soil).

Particle size analysis of the soil sample showed that approximately $95 \%$ of the particles had a diameter in the $1-10 \mu \mathrm{m}$ range, the remaining $5 \%$ had diameters up to $200 \mu \mathrm{m}$.

Carrier for slurry measurements: $0.1 \% \quad(\mathrm{v} / \mathrm{v})$ Triton X-100 solution. A Dye sample of $0.1 \mathrm{mg} \mathrm{ml}^{-1}$ was prepared by dissolving bromocresol green in a $0.01 \mathrm{M}$ disodium tetraborate solution. The carrier for the Dye samples was $0.01 \mathrm{M}$ sodium tetraborate solution.

\subsection{Slurry procedure}

Fig. 1 also shows the sample vessel. $20 \mathrm{ml}$ of a slurry sample was stirred vigorously for $10 \mathrm{~min}$ in a Glass vessel $(50 \mathrm{~mm}$ in height and $23 \mathrm{~mm}$ in diameter). After this the magnetic stirrer was set at a medium speed (400 rpm). Collection of sample took place through $0.75 \mathrm{~mm}$ i.d. (1.6 $\mathrm{mm}$ o.d.) PTFE tubing with the inlet positioned $1 \mathrm{~cm}$ above the bottom of the vessel. During the load cycle the sample loop was rinsed and filled. Propulsion of the sample with $1 \mathrm{~atm}$ (nitrogen) gas pressure resulted in a relatively high flow rate of approximately $30 \mathrm{ml} \mathrm{min}{ }^{-1}$ and the total load cycle took $5 \mathrm{~s}$.

\section{Results and discussion}

\subsection{Straight tube}

\subsubsection{Dye and slurry sample injections}

Table 1 shows the results of the straight horizontal tube for Dye and slurry sample injections at carrier flow rates of 4,5 and $6 \mathrm{ml} \mathrm{min}^{-1}$. A run consisted of four successive injections out of the same sample vessel. For each run the mean of the first moment $\left(M_{1}\right)$ and the mean of the square root of the second moment $\left(\sqrt{M_{2}}\right)$ were calculated, these are depicted in Table 1. For each run the relative standard deviation (r.s.d.) of $M_{1}$ and $\sqrt{M_{2}}$ was calculated as well. The square root of the second moment characterizes the width of the response peak, therefore the time interval $M_{1} M_{1} \pm \sqrt{M_{2}}$ is an indication for the variation in residence time of a sample plug. Fig. 2 shows typical response peaks of Dye, Dowex and Glass injections at

Table 1

Statistical moments for different flow rates; straight tube

\begin{tabular}{|c|c|c|c|c|c|c|}
\hline \multirow[t]{3}{*}{ Sample } & \multicolumn{6}{|c|}{ Flow rate $\left(\mathrm{ml} \mathrm{min}^{-1}\right)$} \\
\hline & \multicolumn{2}{|l|}{4} & \multicolumn{2}{|l|}{5} & \multicolumn{2}{|l|}{6} \\
\hline & $M_{1}$ & $\sqrt{M_{2}}$ & $M_{1}$ & $\sqrt{M_{2}}$ & $M_{1}$ & $\sqrt{M_{2}}$ \\
\hline Dye & 43.4 & 16.4 & 34.5 & 13.4 & 29.9 & 12.3 \\
\hline Dowex & 48.3 & 17 & 36.6 & 4.1 & 30.5 & 3.6 \\
\hline Glass & & & 96 & 18.7 & 72.6 & 17 \\
\hline Soil & & & 36.9 & 15 & 29.1 & 10 \\
\hline
\end{tabular}

All $M_{1}$ and $\sqrt{M_{2}}$, r.s.d. $<3 \%(n=4)$, except for the Glass and soil samples (r.s.d. 5-10\%). 


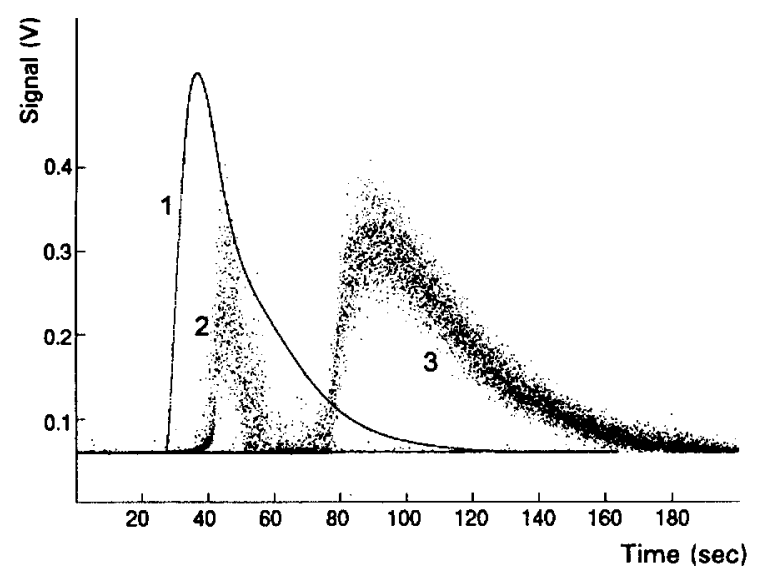

Fig. 2. Typical response peaks. Straight tube, flow rate: $5 \mathrm{ml} \mathrm{min}{ }^{-1}$; injection at $\mathrm{t}=10 \mathrm{~s}$. 1 , Dye; 2 , Dowex; 3, Glass.

a flow rate of $5 \mathrm{ml} \mathrm{min}{ }^{-1}$. The discrete nature of particles causes the 'noisy' slurry peaks [8].

For the Dye injections, $M_{1}$ closely resembles the mean residence time of the carrier, and $\sqrt{M_{2}}$ is relatively large. Both these results were as could be expected [14]. Dowex particles lag behind the mean velocity a little, and it was observed that for the flow rates of 5 and $6 \mathrm{ml} \mathrm{min}^{-1} \sqrt{M_{2}}$ is small (i.e. a small peak width), so the injected plug experiences only limited dispersion. The r.s.d. of $M_{1}$ and $\sqrt{M_{2}}$ for the runs with both Dye and Dowex injections are smaller than 3\%. Glass particles are moving much slower than the average carrier velocity and $\sqrt{M_{2}}$ is large compared to Dye and Dowex. At the flow rate of $4 \mathrm{ml} \mathrm{min}{ }^{-1}$ part of the Glass particles didn't elute within the run time interval which was set at $300 \mathrm{~s}$. The same was true for the Soil sample at $4 \mathrm{ml} \mathrm{min}^{-1}$, at higher flow rates this sample behaved almost the same as the Dye sample. The reproducibility of the Glass and Soil injections, indicated by the r.s.d. of $M_{1}$ and $\sqrt{M_{2}}$, was less than for the Dye and Dowex injections.

\subsubsection{Experimental versus theoretical retention behaviour of dowex and glass particles}

Like in Field Flow Fractionation the retention ratio of solid spheres depends on their size and density [9]. The retention ratio is the ratio of the void time and residence time of the particle $\left(R=t_{0} / t_{r}\right)$. The experi- mentally obtained retention ratio was compared with the expected retention ratio, the latter was calculated using Eq. (12) and Eq. (4).

Fig. 3(A) and (B) show the results for Dowex particles with diameters of 35 and $20 \mu \mathrm{m}$ for several flow rates. Fig. 3(C) and 3(D) show the results for Glass particles of 35 and $17 \mu \mathrm{m}$, respectively. Particles were collected just after the detector during time intervals of approximately 2 to $4 \mathrm{~s}$, depending on the flow rate. For such a time interval the mean particle diameter was determined roughly by using a microscope. For both Dowex and Glass two particle diameters were monitored. This method was not very sophisticated but gave a good impression as can be seen in Fig. 3. The variability of the experimental data, indicated by the error bars in Fig. 3, is mainly due to the monitoring method. Both theoretically and experimentally particles of different sizes and densities, propelled by equal flow rates, clearly have different retention ratios. Therefore, slurry samples containing particles of different sizes and densities will experience relatively large $\sqrt{M_{2}}$ values, so if straight tubes are used as pretreatment manifolds a uniform treatment will be hindered. Furthermore, except for Dowex particles at very low flow rates, particles are more retained than predicted. Part of the differences can be explained by the fact that experimental conditions do not exactly conform the theoretical conditions. Particle diameters are too large compared to the tube diameter and due to the use of a peristaltic pump the flow is not exactly pulse free.

\subsection{Helically coiled tubes}

Two helically coiled tubes with aspect ratios $\lambda$ $=0.75 / 11$ (coil 1) and $\lambda=0.75 / 74\left(\mathrm{~mm} \mathrm{~mm}^{-1}\right)$ (coil 2) were used, both in a horizontal and a vertical orientation. As described before, an experimental run consisted of four injections and $M_{1}$ and $\sqrt{M_{2}}$ were calculated to give an indication for the variation in residence time. Table 2 and 3 show the results for the Dye and slurry injections. The r.s.d. of $M_{1}$ and $\sqrt{M_{2}}$ for these runs were all smaller than $2 \%$, this indicates that the injections and the flow behaviour of the slurry samples had a good reproducibility. Fig. 4 shows typical response peaks of Dye, Dowex and Glass injections. 

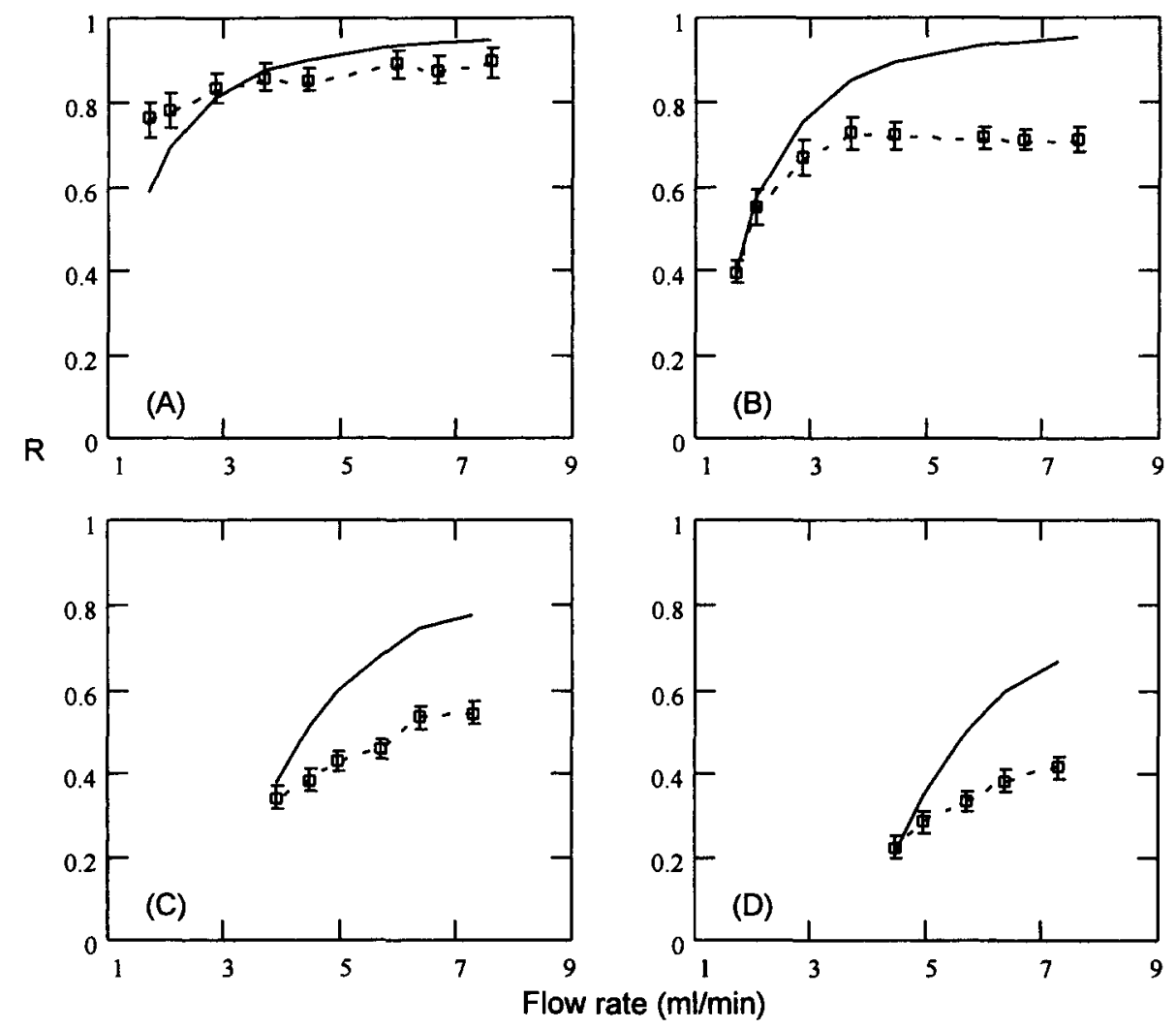

Fig. 3. Horizontal straight tube. Boxes indicate experimental results, solid lines indicate theoretical results. $\mathrm{R}$ is the retention ratio. (A)Dowex particles $(35 \mu \mathrm{m})$; (B)-Dowex $(20 \mu \mathrm{m})$; (C)-Glass $(35 \mu \mathrm{m})$; (D)-Glass $(17 \mu \mathrm{m})$.

Table 2

Statistical moments for different flow rates; helically coiled tube, aspect ratio $\lambda=0.75 / 11$ (coil 1 )

\begin{tabular}{|c|c|c|c|c|c|c|}
\hline \multirow[t]{3}{*}{ Sample } & \multicolumn{6}{|c|}{ Flow rate $\left(\mathrm{ml} \mathrm{min}{ }^{-1}\right)$} \\
\hline & \multicolumn{2}{|l|}{4} & \multicolumn{2}{|l|}{5} & \multicolumn{2}{|l|}{6} \\
\hline & $M_{1}$ & $\sqrt{M_{2}}$ & $M_{1}$ & $\sqrt{M_{2}}$ & $M_{1}$ & $\sqrt{M_{2}}$ \\
\hline Dye & 43.9 & 11.2 & 35.3 & 9.4 & 29.7 & 7.9 \\
\hline Dowex(h) & 36.5 & 3 & 29.7 & 2.3 & 25.2 & 2 \\
\hline Glass(h) & 50.7 & 4.2 & 39.9 & 2.7 & 31.8 & 2.1 \\
\hline Soil(h) & 41 & 8 & 35.2 & 7.5 & 29.4 & 7.1 \\
\hline Donex(v) & 36.9 & 3.1 & 30 & 2.4 & 25.2 & 2.2 \\
\hline Glass(v) & 52.8 & 3.6 & 41.2 & 2.7 & 33.7 & 2.1 \\
\hline Soil(v) & 41 & 8 & 34.3 & 7.7 & 29.1 & 7 \\
\hline
\end{tabular}

$\mathrm{h}$ : horizontal orientation of the coil $v$ : vertical orientation of the coil

For all $M_{1}$ and $\sqrt{M_{2}}$, r.s.d. $<2 \%(n=4)$
Table 3

Statistical moments for different flow rates; helically coiled tube, aspect ratio $\lambda=0.75 / 74$ (coil 2)

\begin{tabular}{|c|c|c|c|c|c|c|}
\hline \multirow[t]{3}{*}{ Sample } & \multicolumn{6}{|c|}{ Flow rate $\left(\mathrm{ml} \mathrm{min}^{-1}\right)$} \\
\hline & \multicolumn{2}{|l|}{4} & \multicolumn{2}{|l|}{5} & \multicolumn{2}{|l|}{6} \\
\hline & $M_{1}$ & $\sqrt{M_{2}}$ & $M_{1}$ & $\sqrt{M_{2}}$ & $M_{1}$ & $\sqrt{M_{2}}$ \\
\hline Dye & 43.4 & 11.2 & 34.8 & 9.3 & 29.3 & 8.2 \\
\hline Dowex (h) & 33.3 & 3.5 & 26.6 & 3.3 & 22.5 & 3.2 \\
\hline Glass(h) & 40.5 & 3.9 & 31.2 & 2.7 & 26.3 & 2.1 \\
\hline Soil(h) & 40.6 & 10.7 & 35.4 & 9.1 & 28.4 & 6.2 \\
\hline Donex(v) & 34.5 & 3.4 & 27.3 & 3.4 & 22.9 & 2.9 \\
\hline Glass(v) & 92 & 24 & 36.2 & 3.6 & 28.9 & 2.5 \\
\hline Soil(v) & 40.9 & 10 & 36 & 9.5 & 29.8 & 7.1 \\
\hline
\end{tabular}

$h$ : horizontal orientation of the coil $v$ : vertical orientation of the coil

For all $M_{1}$ and $\sqrt{M_{2}}$, r.s.d. $<2 \%(n=4)$ 


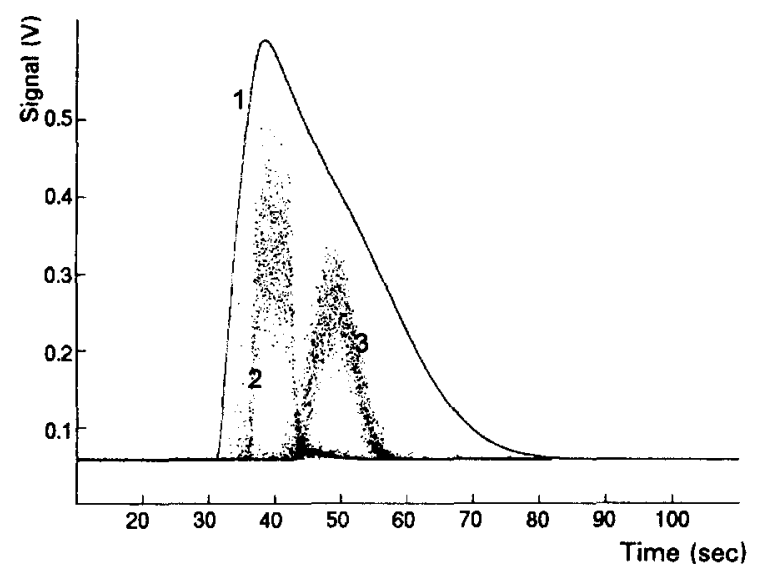

Fig. 4. Typical response peaks, Coil 1, horizontal orientation, flow rate $5 \mathrm{ml} \mathrm{min}^{-1}$, injection at $\mathrm{t}=10 \mathrm{~s}$. 1, Dye; 2, Dowex; 3 , Glass.

\subsubsection{Dye and soil sample injections}

For the Dye injections the orientation of a helically coiled tube had no significant effect on the outcome of the moments. The first moment closely resembled the mean residence time of the carrier and $\sqrt{M_{2}}$ is relatively small compared to the straight tube experiments because secondary flow promotes radial mixing [14].

The main part of the soil sample consisted of small suspended particles, therefore only a small difference in the first moment between the soil sample and the Dye was to be expected. The larger particles only affect the magnitude of the moments (especially of the second moment) if they are strongly retained as in the case of straight tubes. In the coil experiments these larger particles were retained only to a minor extent, therefore the $\sqrt{M_{2}}$ of the soil experiments is smaller compared to the straight tube values.

\subsubsection{Dowex and glass sample injections}

Fig. 5 gives a graphical representation of the results for Dowex and Glass with the Dye injections used as a reference. The bars represent the time interval of the mean residence time $\left(M_{1}\right)$ plus and minus $\sqrt{M_{2}}$. In general, the Dowex and Glass samples have a relatively small $\sqrt{M_{2}}$ compared to the Dye injections and also compared to the straight tube experiments. The small $\sqrt{M_{2}}$ indicates a limited dispersion. For all experiments there was a clear difference between the $M_{1}$ of Dowex and the $M_{1}$ of Glass, so there is a distinct dependence of the particle density on the mean residence time.

\subsubsection{Effect of the aspect ratio}

As already mentioned, both the Dowex and the Glass samples experience only a limited dispersion. The second moments of the experiments with coil 1 (Fig. 5(A) and (B)) are somewhat smaller compared to coil 2 (Fig. 5(C) and 5(D)). Coil 1 shows a clear difference in $M_{1}$ between Dowex and Glass. The Dowex samples have a small $M_{1}$ compared to the mean residence time of the Dye sample, whereas the Glass samples are more retained.

For coil 2 the $M_{1}$ of Dowex is also smaller than the $M_{1}$ of Dye, but even smaller compared to Dowex in coil 1. The $M_{1}$ of Glass is smaller than the $M_{1}$ of Dye as well (except for small flow rates in the vertical orientation). For coil 2 the difference between the $M_{1}$ of Dowex and the $M_{1}$ of Glass is much smaller than the difference between the first moments of coil 1. However, despite this smaller difference, Dowex and Glass still have significantly different residence times.

4.2.3.1. Effect of the coil orientation. The moments for coil 1 in horizontal and in vertical position (5(A) and 5(B)) are quite similar. The orientation hardly affects the Dowex moments and seems to have only a small effect on the retention of the Glass particles. Therefore it can be concluded that, for this relatively large aspect ratio, the gravitational force is considerably less important compared to the forces induced by the secondary flow. The horizontal oriented coil with the small aspect ratio (coil 2, Fig. 5(C)) shows the least separation between the Dowex and the Glass particles. As the results show in the Fig. 5(C) and (D), the orientation of coil 2 has little effect on the moments of Dowex. However, for the Glass particles clearly the first moment increases in the case of vertical orientation, especially if the flow rate decreases (the result for the $4 \mathrm{ml} \mathrm{min}^{-1}$ flow can be extracted from Table 3). The gravitational force is becoming more important if the aspect ratio decreases and the density of the particles increases. 


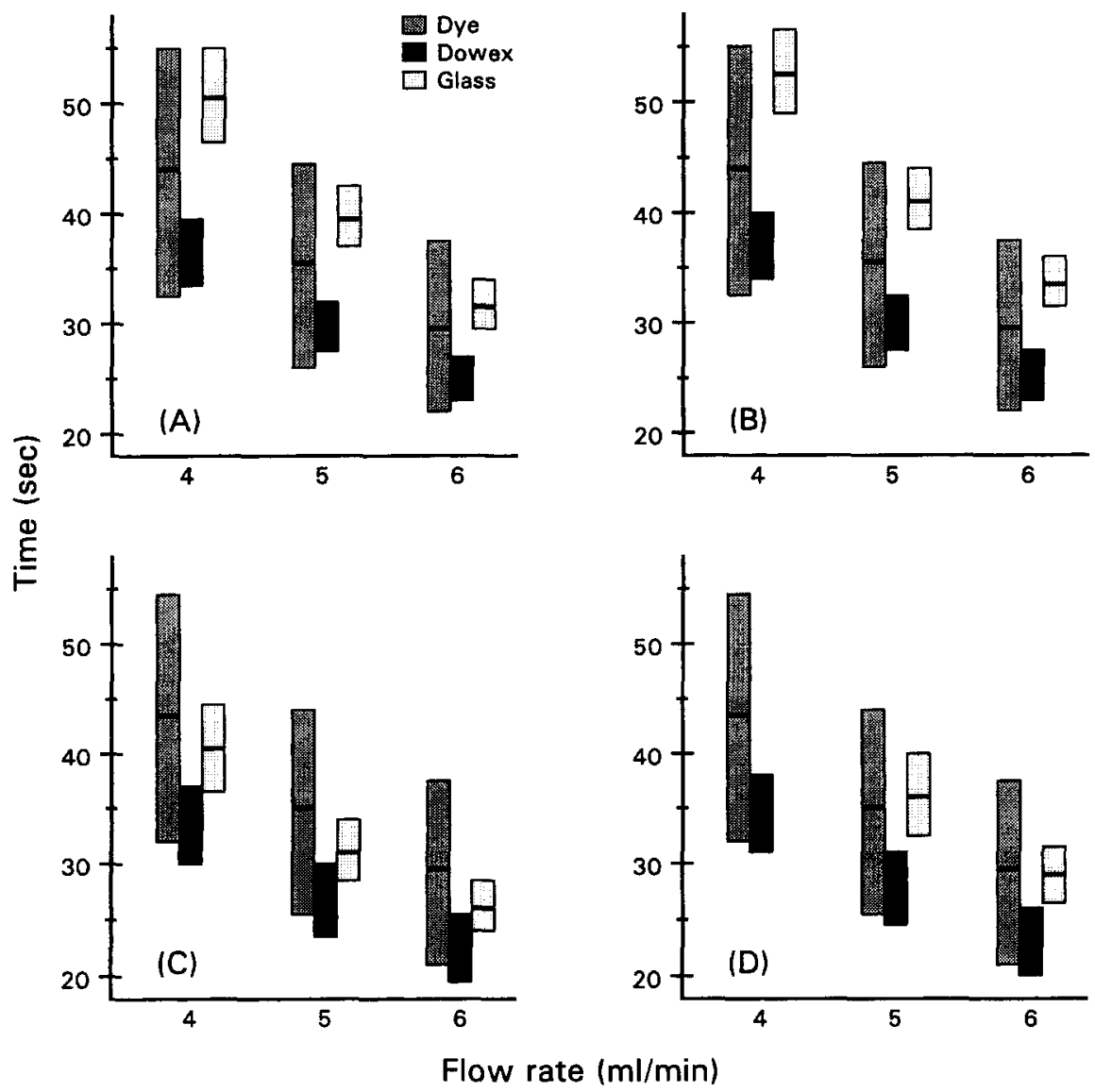

Fig. 5. Residence times of Dye and slurry sample for helically coiled tubes. The dash in the middle of a bar represents $M_{1}$ and the position of the bar indicates the time interval $M_{1} \pm \sqrt{M_{2}}$. (A) coil 1 horizontal orientation; (B) 1 vertical; (C) coil 2 horizontal; (D) coil 2 vertical. coil 1: $\lambda=0.75 / 11\left(\mathrm{~mm} \mathrm{~mm}^{-1}\right)$; coil 2: $\lambda=0.75 / 74\left(\mathrm{~mm} \mathrm{~mm}^{-1}\right)$.

\section{Conclusion}

In order to achieve a reproducible sample pretreatment a small $\sqrt{M_{2}}$ of the slurry plug is needed. For a slurry sample with variations in particle density, a coil with a small aspect ratio and a horizontal orientation can be recommended. For slurry samples containing particles with equal densities a tightly coiled tube (large aspect ratio) will ensure a small $\sqrt{M_{2}}$ along with a large $M_{1}$.

The theoretical relations gave a reasonable impression of the retention ratios for straight tube experiments. For coils an empirical approach seems to be more appropriate. Therefore more experiments will be performed in the near future.

\section{References}

[1] S.J. Haswell and D. Barclay, Analyst, 117 (1992) 117.

[2] M. de la Guardia, V. Carbonell, A. Morales-Rubio and A. Salvador, Talanta, 40 (1993) 1609.

[3] R. Martinez-Avila, V. Carbonell, A. Salvador and M. de la Guardia, Talanta, 40 (1993) 107.

[4] T. Guo and J. Baasner, Talanta, 40 (1993) 1927.

[5] P. Vinas, N. Campillo, I. Lopez Garcia and M. HernandezCordoba, Anal. Chim. Acta, 283 (1993) 393.

[6] A. Morales-Rubio, M.L. Mena and C.W. McLeod, Anal. Chim. Acta, 308 (1995) 364.

[7] P. Torres, E. Ballesteros and M.D. Luque de Castro, Anal. Chim. Acta, 308 (1995) 371.

[8] M. Hulsman, M. Bos and W.E. van der Linden, Anal. Chim. Acta, 324 (1996) 13.

[9] J. Janca, Field Flow Fractionation, Dekker, New York, 1988. 
[10] G. Stegeman, Ph.D. Thesis, University of Amsterdam, Amsterdam, 1994.

[11] D.W. Morton and C.L. Young, J. of Chrom. Sci., 33 (1995) 514.

[12] S.D. Kolev, Anal. Chim. Acta, 308 (1995) 36.

[13] B.F. Johnson, R.E. Malick and J.G. Dorsey, Talanta, 39 (1992) 35 .

[14] J. Ruzicka and E.H. Hansen, Flow Injection Analysis, 2nd edn., Wiley, New York, 1988, p. 23-36.

[15] S.H. Brooks, D.V. Leff, M.A. Hernandez Torres and J.G. Dorsey, Anal. Chem., 60 (1988) 2737.
[16] A. Savitsky and M.J.E. Golay, Anal. Chem., 36 (1964) 1627.

[17] J.G. DosRamos and C.A. Silebi, J. Colloid Interface Sci., 133 (1989) 302.

[18] G. Green and G. Belfort, Desalination, 35 (1980) 129.

[19] G. Belfort and N. Nagata, Desalination, 53 (1985) 57.

[20] P.S. Williams, S. Lee and J.C. Giddings, Chem. Eng. Comm., 130 (1994) 143.

[21] R.S. MacTaggart, H.S. Nasr-El-Din and J.H. Masliyah, Chem. Eng. Sci., 48 (1993) 921.

[22] P.J. Worsfold and J.R. Clinch, Anal. Chim. Acta, 197 (1987) 117. 